Case Report

\title{
Mathematical Modelling of Epidemiology of Malaria: A Case Study of Luapula Province of Zambia
}

\author{
Justina Mulenga, Leonard Mubila \\ Department of Mathematics, School of Mathematics and Natural Sciences, The Copperbelt University, Kitwe, Zambia
}

Email address:

justinamwenya@gmail.com (J. Mulenga),mubilalxm@gmail.com (L. Mubila)

\section{To cite this article:}

Justina Mulenga, Leonard Mubila. Mathematical Modelling of Epidemiology of Malaria: A Case Study of Luapula Province of Zambia. American Journal of Applied Mathematics. Vol. 4, No. 6, 2016, pp. 289-295. doi: 10.11648/j.ajam.20160406.15

Received: March 10, 2016; Accepted: March 18, 2016; Published: November 25, 2016

\begin{abstract}
In this study, we use the SIR model proposed by Kermack and McKendrick to model the epidemiology of malaria in Luapula Province. Data collected from the District Health Management Teams in Luapula Province were used to analyse the rate of infection of malaria in the Province. The Reproduction number $R_{0}$, was calculated and it was found if $R_{0}>0$, there will be malaria outbreak in the province and if $R_{0}<0$, the disease will not evade the Province. From our analysis we found $R_{0}>0$ which implies that the force of malaria infection in the Province is high. We, therefore, make recommendations for the reduction of malaria in the Province.
\end{abstract}

Keywords: SIR, Reproduction Number, Malaria, Luapula Province

\section{Introduction}

Malaria is an ancient disease which has significantly caused huge social, economic and health burdens. Its prevalence is mainly in tropical countries. The disease has been investigated for many years but still remains a major public health problem with 109 countries declared as endemic to the disease by 2008 (Johansson and Leander, 2010). A worldwide estimated number of malaria cases in the year 2010 was 219 million resulting in 660000 deaths (World Health Organization (WHO), 2012). About $91 \%$ of the deaths were in African region, $6 \%$ in South-East Asian region and 3\% in the Eastern Mediterranean Region. The majority of deaths (86\%) were children (Centre for Disease Control and Prevention (CDC), 2013).

Malaria is an infectious disease which is transmitted to the humans through the bites of the female anopheles mosquitoes. The mosquitoes contain parasites called plasmodium. There are four types of plasmodium parasites namely; plasmodium falciparum, plasmodium vivax, plasmodium ovale and plasmodium malariae. Plasmodium falciparum is responsible for about three-quarters of reported malaria cases, Lalloo et al., (2007). The successful development of the malaria parasite within the mosquito depends on factors such as ambient temperature and humidity, and also whether the mosquito survives long enough to enhance the completion of the life cycle of the parasite within the mosquito host (Ngwa\& Shu, 2000).

Malaria presents a significant public health problem in much of the developing world regardless of huge efforts that have been put in the last century to eradicate or control it. It remains the most prevalent and devastating parasitic disease in the tropics with $80 \%$ prevalence being in the sub-Saharan Africa. Despite much of the control of the disease through drug treatment, it can also be controlled by other preventive health measures. Risking developing severe acquired immunity tends to be location specific. In highly endemic areas the risk is to young children and immigrants and travelers from non-endemic areas. Another group that is prone to malaria infection is the pregnant women whose natural immunity is depressed during pregnancy.

Some control strategies such as intermittent preventive treatment (IPT) for pregnant women and infants have widely been used (Musekwa, 2008). This is because of the fact that treatment using drugs and immune mechanism act synergically to clear infection. Other malaria control interventions that reduce human-vector contact are widely used in areas that are infected with malaria. These include the use of bed nets treated with insecticide and screened houses that reduce human-vector contact. With no effective vaccine 
in sight and vast older antimalarial drugs drastically losing effectiveness due to drug resistance developed by the parasite, prevention is the only advisory given to the affected people.

Malaria is endemic in Zambia and has geographic and seasonal variations. A report from the Zambia Demographic and Health survey 2007 indicates that 4.3 million cases of malaria (including confirmed and unconfirmed cases) were reported in the country resulting in 6149 deaths (Central Statistical Office (CSO), Ministry Of Health $(\mathrm{MOH})$ and Micro International Inc, 2008). The annual incidence rate of malaria was estimated at 358 cases per 1000 population in the year 2007 which showed a drop from 412 cases per 1000 population recorded in the year 2006 (CSO,MOH and Micro International Inc, 2008). High disease prevalence rate have been recorded in the Northern, Luapula and Eastern provinces while Lusaka province had the lowest malaria incidence rate. The Health Survey 2007 further reports that malaria accounts for up to 40 percent of all infant mortality and 20 percent of maternal mortality in Zambia. Therefore, malaria poses a critical social and economic burden on communities living in areas that are endemic to the disease.

Areas in which there is endemic malaria have various problems, some of which are daily increase of the malaria infection rate. The government spends huge amount of money for treatment during an outbreak of malaria. The endemic eventually make people fail to go for work thereby lowering productivity of the economy. Due to insufficient preventive measures and treatment, the disease leads to untimely death.

Even though malaria has been investigated for hundreds of years, it still remains a major health problem. The prominent researchers who have carried out investigations on malaria include Aron and May (1982), Bailey (1982), Ross (1909), Anderson and May (1989) and Tumwiine et al. (2007). It is in light of the above that this study is carried out to look at mathematical modeling of malaria: a case study of Luapula Province of Zambia. This study is of great importance to Luapula Province.

\section{Mathematical Model}

We use the SIR model which describes the different states which a human can be in. There are three stages in this model which are Susceptible, Infectious and Removed. A human moves through different states at different rates. Humans enter the system in the susceptible state when born at rate $\Pi$. A susceptible human enters the infectious state at rate $\gamma$ when receiving the disease. From the infected state, individuals can either move to recovered at the rate $v$ or they can leave the system by disease induced death at the rate $\sigma$. Humans can also leave the system by natural death at rate $\mu$. The total population is denoted as $\mathrm{N}$. The interaction between the states of this model is illustrated in figure 1.

Compartmental diagram

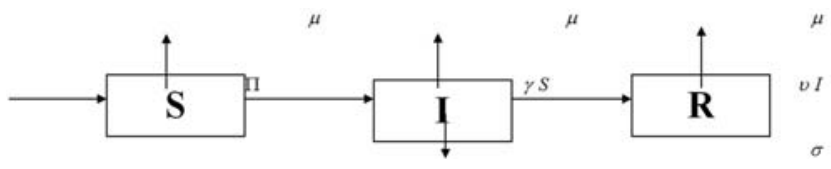

Figure 1. The basic SIR model with rates $\Pi, \mu, \gamma, v$ and $\sigma$

\section{Model Equations}

From the compartmentalized model, we have a set of ordinary differential equations describing the model. The resulting equations are:

$$
\begin{gathered}
\frac{d S}{d t}=\Pi-\gamma S I-\mu S \\
\frac{d I}{d t}=\gamma S I-I(\mu+\sigma+v) \\
\frac{d R}{d t}=v I-\mu R
\end{gathered}
$$

\section{Model Assumptions}

- The recovered humans have immunity to the disease.

- There is homogeneous interaction in the population.

- Birth rates and natural death rates are constant

Existence of Equilibrium States of the SIR Model

Here, we present the results of the existence of equilibrium points of the SIR model

Equilibrium Analysis

At equilibrium: $\frac{d S}{d t}=\frac{d I}{d t}=\frac{d R}{d t}=0$

Equations (1) - (3) become

$$
\begin{aligned}
\Pi-\gamma S I-\mu S & =0 \\
\gamma S I-I(\mu+\sigma+v) & =0 \\
v I-\mu R & =0
\end{aligned}
$$

The incidence rate $(G)$ in the above model, the function $G=\gamma S I$ models the transmission rate from the compartment of susceptible individuals to the compartment of infectious individuals. This is the incidence rate of malaria parasite infection on the population. Here we consider incidence rate e, $\mathrm{g}$ infection depends on the fraction of infectious since the population is large. Thus $G=\frac{\gamma I S}{N}$

The basic reproduction number $\left(R_{O}\right)$

$$
R_{O}=\frac{\gamma}{\mu+\sigma+v}
$$

which is the number of infections from a single infection in a population where all subjects are susceptible. From equation (2),

$$
\begin{aligned}
\frac{d I}{d t} & =\gamma S I-I(\mu+\sigma+v) \\
& =\frac{\gamma}{(\mu+\sigma+v)} \cdot(\mu+\sigma+v) S I-I(\mu+\sigma+v) \\
& =R_{O}(\mu+\sigma+v) S I-I(\mu+\sigma+v)
\end{aligned}
$$




$$
\begin{aligned}
& =[I(\mu+\sigma+v)]\left[R_{O} S-1\right] \\
& =\left[R_{O} S-1\right][(\mu+\sigma+v) I] \\
& =\left(R_{O} S-1\right)(\mu+\sigma+v) I
\end{aligned}
$$

It yields that for $R_{O}>0$ in equation (7) above:

$$
\frac{d I}{d t}>0
$$

This means that there will be a proper malaria outbreak in the province with an increase in the number of infected individuals in the population. The disease free equilibrium is unstable and the host will not recover from the disease.

Now, if $R_{O}<0$,

This means that the disease can never cause a proper epidemic outbreak. As such the human host will always recover from the disease.

From equation (5)

$$
\begin{aligned}
& \gamma S I-I(\mu+\sigma+v)=0 \\
\Rightarrow & I[\gamma S-(\mu+\sigma+v)]=0 \\
\Rightarrow & I=0 \text { or } \gamma S-(\mu+\sigma+v)=0
\end{aligned}
$$

Substituting $I=0$ in equation (3) we have

$$
\begin{gathered}
-\mu R=0 \\
R=0
\end{gathered}
$$

Using equation (1), we have

$$
\begin{gathered}
\Pi S-\mu S=0 \\
S=\frac{\Pi}{\mu}
\end{gathered}
$$

Thus, the disease free equilibrium is

$$
E_{O}=\left[\frac{\Pi}{\mu}, 0,0\right]
$$

From equation (8), we have

$$
\begin{gathered}
\gamma S-(\mu+\sigma+v)=0 \\
S^{*}=\frac{\mu+\sigma+v}{\gamma}
\end{gathered}
$$

Substituting (9) in equation (4) we get,

$$
\Pi-\gamma S I-\mu S=0
$$

$$
\begin{gathered}
\Pi-\gamma\left(\frac{\mu+\sigma+v}{\gamma}\right) I-\mu\left(\frac{\mu+\sigma+v}{\gamma}\right)=0 \\
(\mu+\sigma+\mu) I=\Pi-\mu\left(\frac{\mu+\sigma+v}{\gamma}\right) \\
I^{*}=\frac{\gamma \Pi-\mu(\mu+\sigma+v)}{\gamma(\mu+\sigma+v)}
\end{gathered}
$$

Substituting equation (10) in equation (6), we get

$$
\begin{gathered}
v I-\mu R=0 \\
v\left(\frac{\gamma \Pi-\mu(\mu+\sigma+v)}{\gamma(\mu+\sigma+v)}\right)-\mu R=0 \\
\frac{v \gamma \Pi-v \mu(\mu+\sigma+v)}{\gamma(\mu+\sigma+v)}-\mu R=0 \\
-\mu R=-\left(\frac{v \gamma \Pi-v \mu(\mu+\sigma+v)}{\gamma(\mu+\sigma+v)}\right) \\
R^{*}=\left[\frac{v \gamma \Pi-v \mu(\mu+\sigma+v)}{\mu \gamma(\mu+\sigma+v)}\right]
\end{gathered}
$$

Thus the endemic equilibrium state is

$$
E_{*}=\left[\frac{\mu+\sigma+v}{\gamma}, \frac{\gamma \Pi-\mu(\mu+\sigma+v)}{\gamma(\mu+\sigma+v)}, \frac{v \gamma \Pi-v \mu(\mu+\sigma+v)}{\gamma \mu(\mu+\sigma+v)}\right]
$$

\section{Stability Analysis}

To establish the equilibrium points, we use the Jacobian matrix of equation system (1), which gives us:

$$
J=\left[\begin{array}{ccc}
-\gamma I-\mu-\lambda & -\gamma S & 0 \\
\gamma I & \gamma S-(\mu+\sigma+v)-\lambda & 0 \\
0 & v & -\mu-\lambda
\end{array}\right]
$$

Where $\lambda$ is the eigen values of $\mathrm{J}$.

$$
\begin{aligned}
\text { Det } \mathrm{J}=(-\gamma I-\mu-\lambda)\left|\begin{array}{cc}
\gamma S-(\mu+\sigma+v)-\lambda & 0 \\
v & -\mu-\lambda
\end{array}\right|+\gamma S \\
\left|\begin{array}{cc}
\gamma I & 0 \\
0 & -\mu-\lambda
\end{array}\right| \\
=(-\gamma I-\mu-\lambda)[(\gamma S-(\mu+\sigma+\sigma)-\lambda)(-\mu-\lambda)] \\
=(-\mu-\lambda)\left[\left(\frac{\gamma \Pi}{\mu}-(\mu+\sigma+v)-\lambda\right)(-\mu-\lambda)\right]
\end{aligned}
$$

Thus, the disease free equilibrium is stable when 
$\frac{\eta \Pi}{\mu}<(\alpha+\sigma+\mu)$ where $\frac{\gamma \Pi}{\mu}$ the number of is infected in the population.

\section{Results and Discussion}

Table 1, shows the data collected from Luapula province. The data is presented as statistically analysis of the districts projected populations for the year 2013.

Table 1. Projected population for 2013 for selected districts in Luapula Province SAMFYA.

\begin{tabular}{ll}
\hline DISTRICT & POPULATION \\
\hline SAMFYA & 194,272 \\
MWENSE & 84,617 \\
KAWAMBWA & 98,806 \\
NCHELENGE & 173,680 \\
MANSA & 212,650 \\
\hline
\end{tabular}

Table 2. Malaria records for Samfya MWENSE.

\begin{tabular}{llll}
\hline YEAR & POPULATION & CASES & PERCENT \\
\hline 2013 & 194272 & 96165 & 50 \\
2012 & 192329 & 88471 & 46 \\
2011 & 190406 & 104914 & 55 \\
2010 & 188502 & 40151 & 21 \\
2009 & 186617 & 38256 & 21 \\
2008 & 184520 & 43591 & 24 \\
\hline
\end{tabular}

Table 3. Malaria records for Mwense KAWAMBWA.

\begin{tabular}{llll}
\hline YEAR & POPULATION & CASES & PERCENT \\
\hline 2013 & 84617 & 65673 & 78 \\
2012 & 83771 & 51912 & 62 \\
2011 & 82933 & 44142 & 53 \\
2010 & 82104 & 40127 & 49 \\
2009 & 81283 & 39570 & 49 \\
2008 & 80470 & 36920 & 46 \\
\hline
\end{tabular}

Table 4. Malaria records for kawambwa NCHELENGE.

\begin{tabular}{llll}
\hline YEAR & POPULATION & CASES & PERCENT \\
\hline 2013 & 98806 & 44463 & 45 \\
2012 & 97818 & 34138 & 35 \\
2011 & 96840 & 46580 & 48 \\
2010 & 95871 & 43430 & 45 \\
2009 & 94913 & 29043 & 31 \\
2008 & 93964 & 27249 & 29 \\
\hline
\end{tabular}

Table 5. Malaria records for Nchelenge MANSA.

\begin{tabular}{llll}
\hline YEAR & POPULATION & CASES & PERCENT \\
\hline 2013 & 173680 & 83366 & 48 \\
2012 & 171943 & 97664 & 57 \\
2011 & 170224 & 76090 & 45 \\
2010 & 168522 & 54264 & 32 \\
2009 & 166836 & 63231 & 38 \\
2008 & 165168 & 50872 & 31 \\
\hline
\end{tabular}

Table 6. Malaria records for mansa COMBINED PROVINCIAL 5 DISTRICT DATA.

\begin{tabular}{llll}
\hline YEAR & POPULATION & CASES & PERCENT \\
\hline 2013 & 212650 & 67835 & 32 \\
2012 & 210524 & 97683 & 46 \\
2011 & 208418 & 121716 & 58 \\
2010 & 206334 & 125864 & 61 \\
2009 & 204271 & 77419 & 38 \\
2008 & 202228 & 58242 & 29 \\
\hline
\end{tabular}

Table 7. Combined cases for five districts.

\begin{tabular}{llll}
\hline YEAR & POPULATION & CASES & PERCENT \\
\hline 2013 & 654370 & 327010 & 50 \\
2012 & 647827 & 333309 & 51 \\
2011 & 641348 & 332670 & 52 \\
2010 & 634935 & 303812 & 48 \\
2009 & 628586 & 248833 & 40 \\
2008 & 622300 & 210203 & 34 \\
\hline
\end{tabular}

Calculations of the basic reproduction ratio with target population 2013

From equation (7)

$$
\begin{gathered}
R_{O} S-1=0 \\
R_{O} * S=1 \\
R_{O}=\frac{1}{S} \\
R_{O} \propto \frac{k}{S(t)}
\end{gathered}
$$

For all $k>0$,

1. $R_{O}$ for Mansa

$$
R_{O}=\frac{k}{\text { Population }}, \text { for } k=0.25,0.5,0.75,1
$$

$R_{O}=0.00000117560 .0000023513,0.0000035269$, 0.0000047026 .

2. $R_{O}$ for Samfya

$$
R_{o}=\frac{k}{\text { Population }}=0.0000051474 k
$$

for $k=0.25,0.5,0.75,1$

$R_{O}=0.0000012869,0.0000025737,0.0000038606$, 0.0000051474 .

3. $R_{O}$ for Mwense

$$
R_{O}=\frac{k}{\text { population }}=0.000011818 k
$$

for $k=0.25,0.5,0.75,1$

$R_{O}=0.0000029545,0.0000059090,0.0000088635$, 0.000011818 . 
4. $R_{O}$ for Kawambwa

$$
R_{O}=\frac{k}{\text { population }}=0.000010121 k
$$

For $k=0.25,0.5,0.75,1$

$R_{O}=0.00000253030 .0000050605,0.0000075906$ 0.000010121

5. $R_{O}$ for Nchelenge

$$
R_{O}=\frac{k}{\text { population }}=0.0000057577 k
$$

For $k=0.25,0.5,0.75,1$

$R_{o}=0.00000143940 .00000287890 .00000431823$ 0.0000057577 .

The following figure show the malaria reproductive ratios for each district.

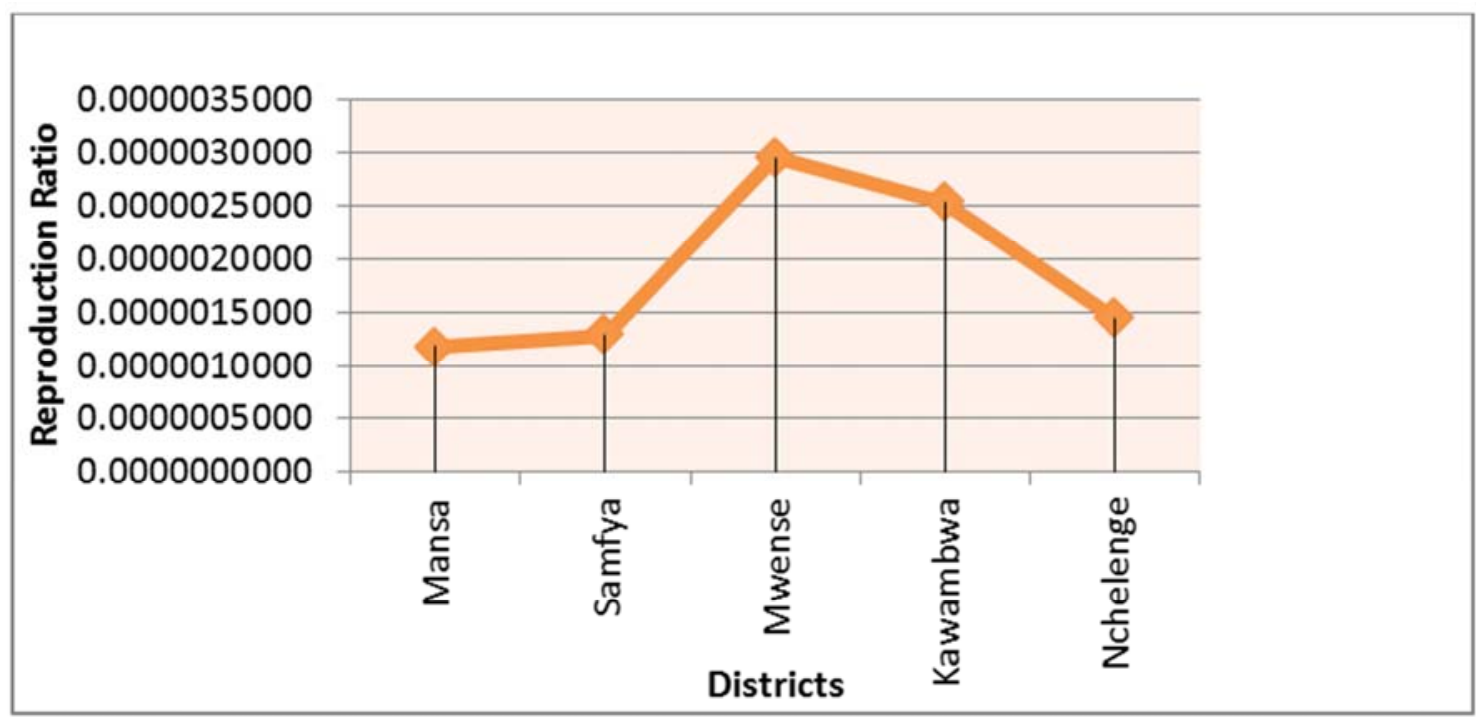

Figure 2. Reproduction Ratio with $k=0.25$.

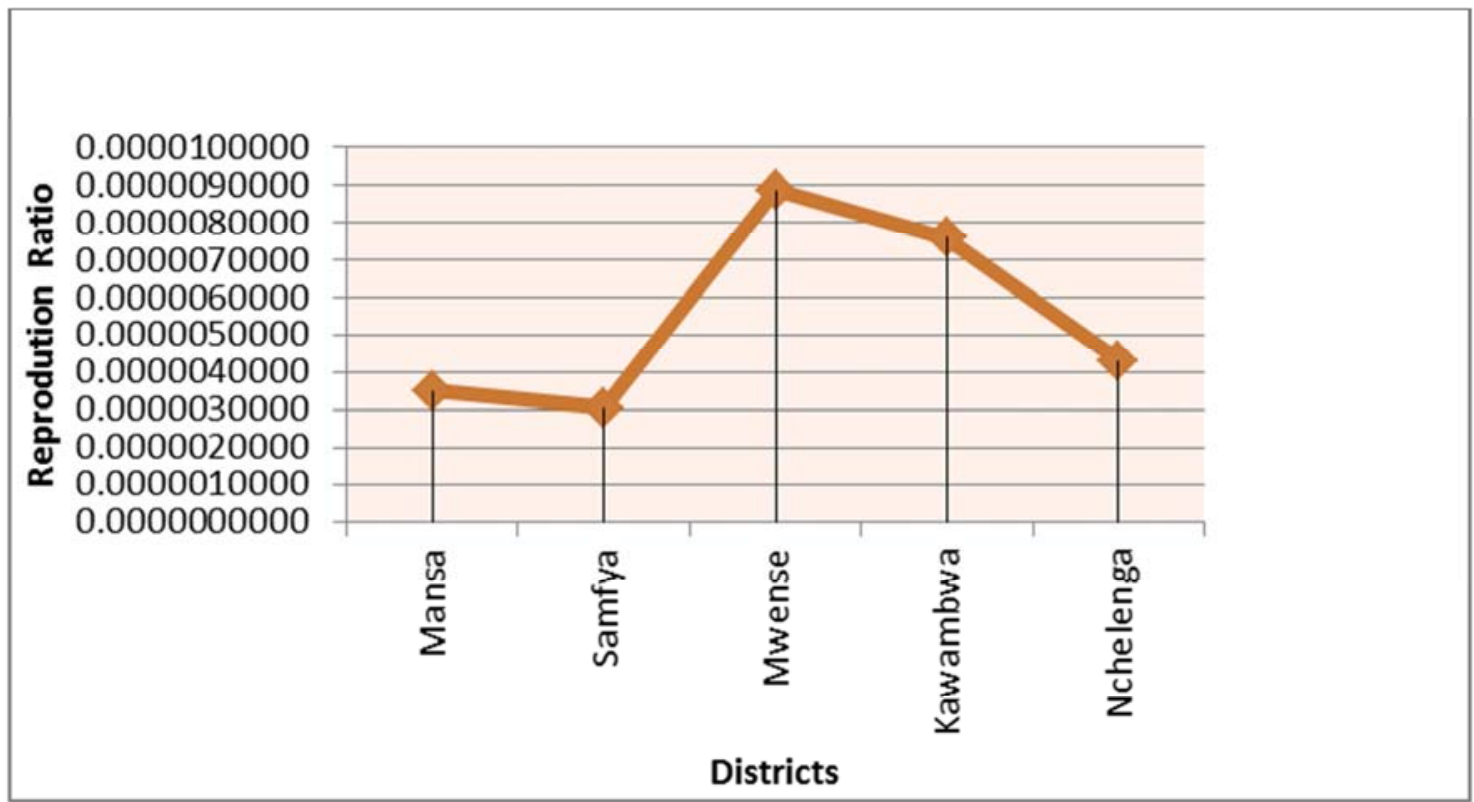

Figure 3. Reproduction Ratio with $k=0.5$. 


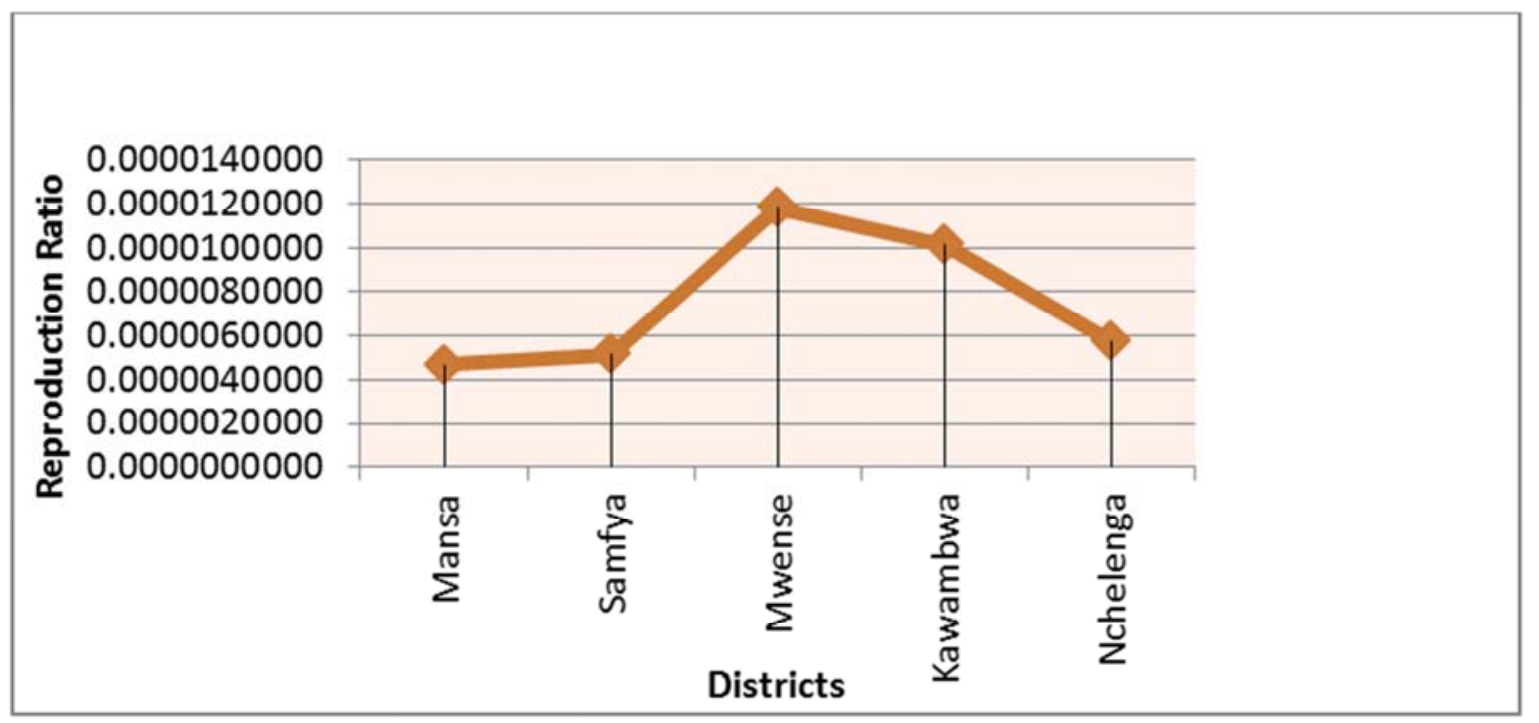

Figure 4. Reproduction Ratio with $k=0.75$.

Malaria SIR Deterministic Model

The general interaction of individuals in a malaria infected area can be outlined as shown in the figure below. The assumption made areInitial values are: $I(0)=0.001, S(0)=0.999, \mathrm{R}(t)=0$,

Parameter values are: $\gamma=0.3, \nu=0.1$.

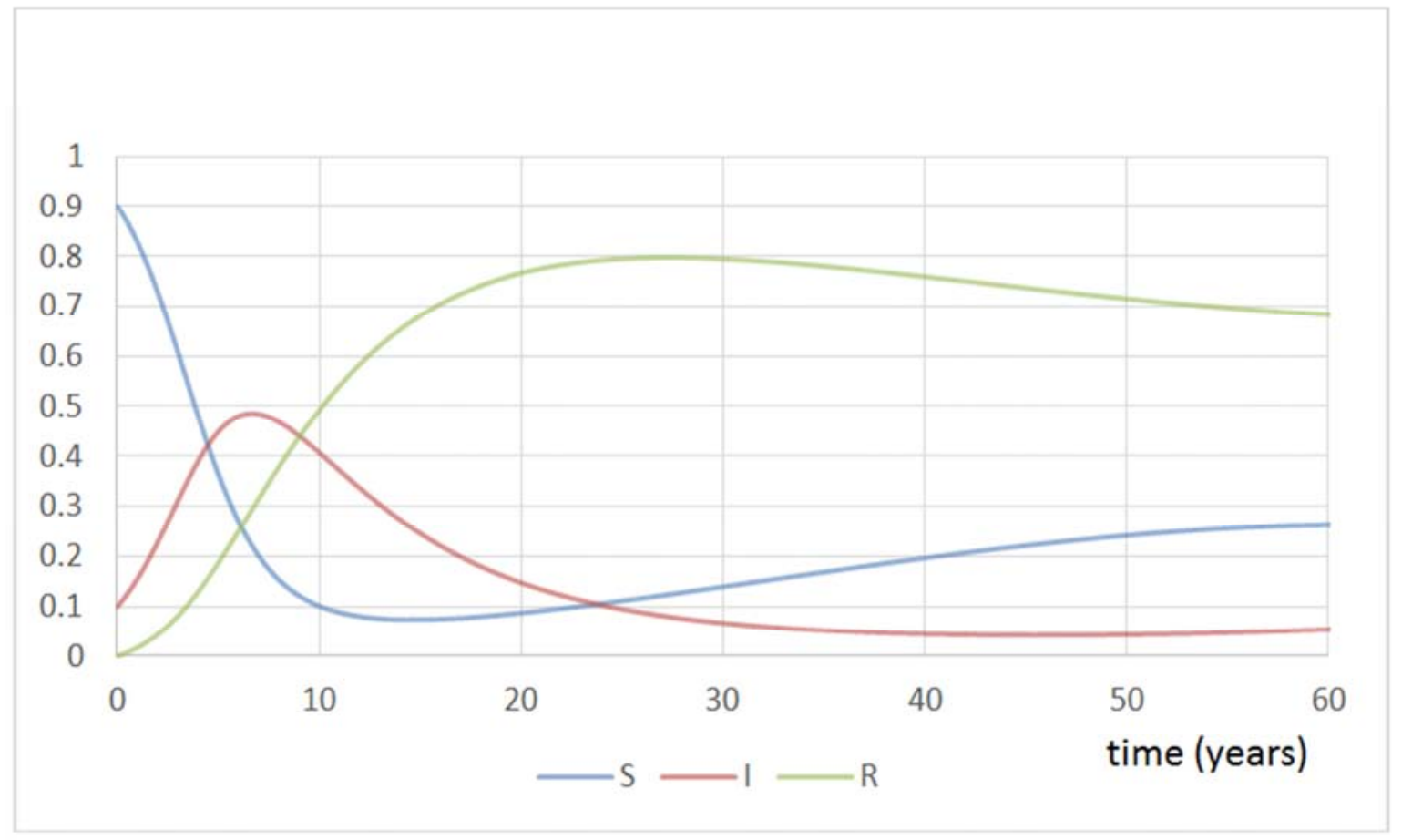

Figure 5. The malaria model.

Kermack - McKendrick. A general epidemic evolves according to the differential equations from an initial values of $\left(S_{0}, I_{0}, R_{0}\right)$ where the total population is $N$. When the infectives are outmately reduced the survival population increase as a positive $S_{\infty}$. The major outbreak of malaria occurs if and only if $\frac{d I}{d t}=0$ meaning that the number of susceptible individuals is also high.

\section{Conclusion}

Model analysis and simulation suggest control strategies should be implemented. Estimates of $R 0$ for malaria in different districts indicate high malaria prevalence thus an immediate need for intervention. The disease has affected vast parts of the population due to prevalence of many water bodies which are a breeding ground for mosquitoes (the malaria vectors). 


\section{Recommendations}

- A need for preventive measures such as distribution of mosquito nets.

- Spray of water bodies to reduce reproduction of mosquitoes can serve as an urgent solution to the disease spread.

- Vaccines against fever should be made available by the government.

\section{Limitation}

The simple SIR model presented here have obvious limitations. They assume that the host population is uniform and homogeneously mixing (in reality, children usually have more adequate contacts per day than adults, different geographic and socio-economic groups have different contact rates, etc.), that there is no latent period of the disease, that there is no dynamics of the host population when the disease is absent, etc. Still, despite these limitations, these models have contributed much to our understanding of the course of real epidemics and the way endemic equilibria can be attained; they can also be useful in obtaining some parameter estimates and between-infections comparisons.

\section{References}

[1] Centre for Disease Control and Prevention (USA)(2012), Impact of Malaria, www.cdc.gov/malaria/malaria_worldwide/impact.html, retrieved on Tuesday $26^{\text {th }}$ November, 2013.

[2] Chitnis. N, (2005) Using Mathematical Models in Controlling the Spread of Malaria. PhD thesis, University of Arizona.

[3] Chitnis, N., Cushing J. M., and Hyman, M (2008) Determining important parameters in the spread of malaria through the sensitivity analysis of a mathematical model. Bulletin of Mathematical Biology, 70:12721296.
[4] Central Statistical Office (CSO), Ministry of Health (MOH), Tropical Diseases Research Centre.

[5] (TDRC), University of Zambia (UNZA) and ORC Macro. (2009). Zambia Demographic andHealth Survey 2007: Calverton, Maryland, USA: Central Statistical Office, Central Board ofHealth, and ORC Macro.

[6] Hove-Musekwa, S. D. (2008). Determining effective spraying periods to control malaria via indoor residual spraying in sub-saharan Africa. Advances in Decision Sciences, 2008.

[7] Johansson P. Leander J., (2010) Mathematical Modeling of Malaria - Methods for Simulation of Epidemics, Chalmers University of Technology, Gothenburg.

[8] King, A. T., Mends-Brew, E., Osei-Frimpong, E., \&Ohene, K. R. (2012). Mathematical model for the control of malaria-Case study: Chorkor polyclinic, Accra, Ghana.

[9] Lalloo, D. G., Shingadia, D., Pasvol, G., Chiodini, P. L., Whitty, C. J., Beeching, N. J., ... \& Bannister, B. A. (2007). UK malaria treatment guidelines. Journal of infection, 54(2), 111-121.

[10] MacDonald. G, (1957) The Epidemiology and Control of Malaria. Oxford UniversityPress, London.

[11] Mandal, S., Sarkar, R. R., \& Sinha, S. (2011). Mathematical models of malaria-a review. Malar J, 10, 202.

[12] Ministry of Health (MOH) [Zambia]. 2008c. National Malaria Control Action Plan 2008. Lusaka,Zambia: Ministry of Health

[13] Ngwa, G. A., \&Shu, W. S. (2000). A mathematical model for endemic malaria with variable human and mosquito populations. Mathematical and computer modelling, 32(7), 747-763.

[14] Tumwiine, J., Mugisha, J. Y. T., \&Luboobi, L. S. (2007). A mathematical model for the dynamics of malaria in a human host and mosquito vector with temporary immunity. Applied mathematics and computation, 189(2), 1953-1965.

[15] World Health Organization (2012), World Malaria Report 2012FACT

SHEETwww.who.int/malaria/publications/world report 2012/ wmr 2012, retrieved on Wednesday $27^{\text {th }}$ November, $201 \overline{3}$. 